\title{
Pulses on Demand in Fibre and Hybrid Lasers
}

\author{
Rok Petkovšek 1,* - Vid Agrež1 - Jaka Petelin ${ }^{1}$ - Luka Černe ${ }^{1}$ - Udo Bünting2 - Boštjan Podobnik ${ }^{3}$ \\ 1University of Ljubljana, Faculty of Mechanical Engineering, Slovenia \\ 2LPKF Laser \& Electronics AG, Germany \\ ${ }^{3}$ LPKF Laser \& Electronics d.o.o., Slovenia
}

This paper presents an investigation of pulse-on-demand operation in fibre and hybrid lasers. Two methods for efficient gain control that enable the generation of laser pulses at arbitrary times with controlled pulse parameters are presented. The method of direct modulation of the pump power in the high-power laser oscillator is shown to generate pulses with a duration in the nanosecond range, with repetition rates varying during operation from a single shot to over $1 \mathrm{MHz}$. An advanced method using a combination of marker and idler seeding a fibre amplifier chain is investigated. Such a system can easily achieve repetition rates of several tens of MHz. The lasers' performances were successfully tested in a real environment on an industrial platform for laser transfer printing. Similar concepts were used for a laser source with ultrashort laser pulses (femtosecond range) on demand by using a mode-locked seed as a source and a solid-state amplifier to achieve high pulse energy and peak power.

Keywords: pulse on demand, fibre lasers, gain control, arbitrary repetition rate

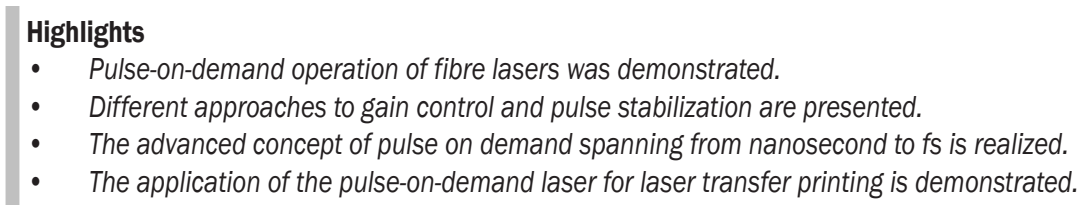

\section{O INTRODUCTION}

Where there is a need for high-precision manufacturing processes, laser-based tools are used. With the demands for increasing production throughput and also for the capability of handling different processes with the same tool, the need for highly flexible laser systems, capable of processing advanced patterns and structures at high speeds, has emerged. This imposes stringent requirements on two key components of a laser processing unit, i.e., the laser-beam scanning system and the laser source. The purpose of the scanning system is to deliver highspeed scanning of the laser beam over the workpiece, while the laser source must meet the requirements for high beam quality, efficiency, ease of maintenance, and rapid laser-power modulation, matching that of the laser-beam scanning system. The latter enables custom pattern generation and precise synchronization with the scanning system.

Fast laser beam steering over the workpiece can be achieved, for example, by using polygon scanners, [1] resonant scanners, [2] or an acousto-optics deflector (AOD) [3] and [4]. An AOD allows precise laser scanning with high resolution but is usually limited to a small working area. For larger working areas and very high scanning speeds, the first two scanning elements are therefore normally used.
State-of-the-art polygon scanners can achieve laser-beam scanning speeds over the workpiece in the range of $\mathrm{km} / \mathrm{s}$; however, they are limited to constant scanning speeds. This means that with conventional laser sources which can only operate at a constant repetition rate, it is only possible to produce equidistant structures on the workpiece. To take advantage of the high scanning speed that the polygon scanners offer and still produce arbitrarily spaced structures, the laser source must be capable of operating at continuously variable repetition rates; thus, generating arbitrary output patterns. Such laser sources are said to deliver pulses-on-demand.

Resonant scanners have a similar disadvantage. The scanning angle and, consequently, the position of the laser beam on the workpiece follows the nonlinear response (sinusoidal oscillation) of the scanner's mirror. This nonlinear response makes it difficult to synchronize the scanning system with the laser source. As previously mentioned, only an advanced laser source capable of delivering pulses-on-demand can overcome this problem.

In the literature, we can find different methods for pulse-on-demand laser operation. Laser sources producing variable pulses on a nanosecond scale are usually based on a continuous wave $(\mathrm{CW})$ or fixed-repetition-rate laser [5] and use an external electro-optical modulator (EOM) or acousto-optical modulator (AOM) [6] to modulate the laser output in 
such a way that the desired pulse shape or pattern is achieved [7]. In some laser-processing applications the output laser head is also moved over a larger workpiece (e.g., batch processing) which can put a limit on the size and weight of the laser head. Also, external modulators are usually polarization-sensitive, have limited damage thresholds and in the case of AOM also limited modulation speed, especially when used in a high-power laser system.

With the development of high-power diode lasers, another approach has been made possible. The direct modulation of high-power laser-diode lasers enables lightweight laser-head designs; however, a major downside is the much lower beam quality, when compared to single-mode or nearly-single-mode (fewmode) lasers.

The high modulation bandwidth of high-power diode lasers that are used for pumping the laser system allows for the gain switching technique [8] to [10] to be used, where we can achieve good control over the pulse-generation dynamics and consequently enable pulse-on-demand operation for nearly single-mode beam quality. The gain-switching technique uses modulation of the pump power (provided by the highpower diode lasers) to select only the first spike of the relaxation oscillations that form a single laser pulse [11]. With precise gain control pulses as short as $28 \mathrm{~ns}$ [12] were generated from a ytterbium-doped fibre laser and the achievable (but not limited to) peak power was over $4 \mathrm{~kW}$ [13]. Even shorter laser pulses [14] were generated by a more complicated core-pumping scheme in a thulium-doped fibre. Using clad pumping at $790 \mathrm{~nm}$, an efficient system [15] can be realized, which also has the benefit of not being susceptible to the photodarkening effect [16], which usually causes problems in highly pumped fibre lasers.

Gain-switched lasers can be designed without any modulators and can work in a wide range of spectral regions depending on the active dopant and the substrate used. Using thulium- and holmium-doped fibres, a gain-switched laser operating at wavelengths up to $2.1 \mu \mathrm{m}$ was realized [17]. On the other hand, using an erbium-doped fluoride fiber [18], pulses at $2.8 \mu \mathrm{m}$ were generated.

The laser pulses generated at arbitrary repetition rates with a duration of several $10 \mathrm{~s}$ of nanoseconds and peak power in the kilowatt range are suitable for applications such as direct colour marking, micromachining, laser-transfer printing, selective photocoagulation, etc. In contrast, such pulses are too long to achieve cold ablation [19], where bursts of picosecond pulses [20] and [21] are used to efficiently ablate the material, supercontinuum generation [22] or applications for which high-speed, high-resolution micromachining is required.

To address this requirement for shorter pulses, an advanced laser setup using the gain control of its amplifier stages can be employed to mitigate the transients in the output pulse train when changing the repetition rate of the laser. The pump power modulation [23] can be used, but it can lead to relatively slow response times and, in some cases, must include an advanced thermal management control for pumpwavelength stabilization [24]. The solution that offers laser-pulse generation with a true pulse-on-demand mode uses a combination of idler and marker sources that seed the laser's amplifier chain. The idler signal keeps the gain of the amplifiers constant between marker pulses [25] to [27]. The benefit of this approach is also that the controlled idler signal prevents the build-up of the amplified spontaneous emission (ASE) and enables the formation of marker pulses at very low repetition rates. At the output of the amplification stages, the idler and marker light must be separated so that only the marker signal is sent to the workpiece.

There are two main techniques for marker and idler separation. One is to use either polarization or wavelength filtering, where the idler and marker have either orthogonal polarization or slightly different wavelengths [28] and the filtering can be achieved by a polarizer or a Bragg grating respectively [26] and [27]. The other technique is to use nonlinear filtering, which is more suitable for applications in which the system generates very short marker pulses while the idler seeds the amplifiers with low-power longduration pulses (or CW power) in between the marker pulses. The nonlinear filtering is then realized at the laser output by second harmonic generation (SHG) [29] and [30].

In this paper, we present a compact (using the already-mentioned gain-switching technique) and an advanced (using marker and idler seeding) laser sources, both capable of producing pulses on demand with the ability to efficiently synchronize with the high-speed polygon and resonant scanners for covering different laser applications. Their capabilities are analysed, and the application of laser transfer printing (LTP) in the form of a laser-induced forward transfer (LIFT) is demonstrated.

\section{METHODS}

The output power of lasers is stable when operated under constant conditions, i.e., either continuous wave operation or operation at a fixed repetition rate. In both cases, a steady state is reached in the laser- 
gain media. Changing the repetition of the laser pulses causes the laser to transit to a new steady state, which can be seen as the output-power fluctuation. Driving the laser in the pulse-on-demand operation mode with long pauses between the output pulses results in the active ion inversion population reaching high levels and consequently the production of giant pulses that can exceed the damage thresholds of the laser's optical elements. In contrast, when switching to high repetition rates, the inversion population in the gain media gradually decreases, and laser pulses with a lower peak power are generated. This effect can also be observed in lasers producing bursts of pulses [31] and can be addressed by reshaping the input seed burst so that the output burst consists of pulses with equal peak powers [32].

In this paper, two methods are presented (Fig. 1) that can efficiently control the level of inversion between the laser pulses and, as such, enable pulseon-demand operation with stable pulse parameters decoupled from the effect of changing the repetition rate. One is the gain control in which the pump power is modulated to generate the desired marker pulses from a high power laser oscillator. The other is using a combination of marker and idler seeding in a MOPA (Master Oscillator Power Amplifier) amplifying a chain pumped with constant power.

\section{Pump modulation}

a)
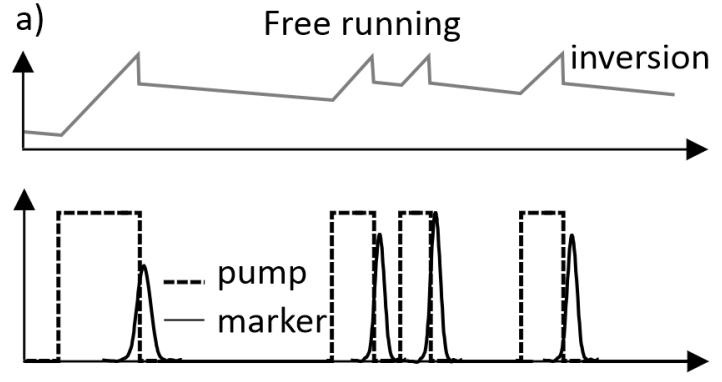

b)
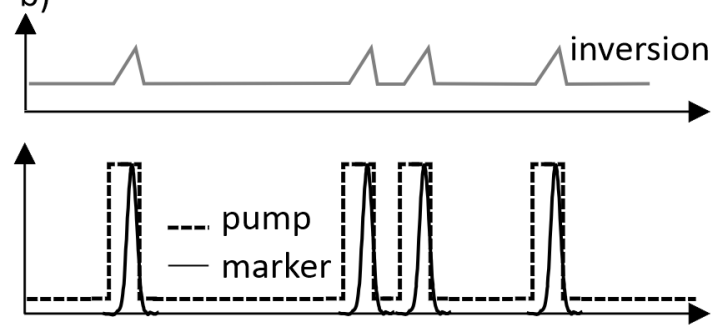

\subsection{Gain Control via Pump-Power Modulation}

Gain control with pump modulation can be used in laser-amplifier chains or in a laser oscillator. The latter is more suitable for high-power oscillator laser systems and is based on the precise pump energy input before the marker pulse formation. The compact system can be realized with the gain-switching technique that can operate over a broad range of repetition rates [33].

The generated pulses are dependent on the pump rate and typically have a ratio of laser peak power to pump peak power equal to ten to one. The duration of the generated laser pulses is dependent on the oscillator's parameters, mainly its length and pump absorption. Combining the oscillator with the pump recovery amplifier [34] enables pulse-duration tuning by at least a factor of two [35].

Upgrading this architecture with an improved pumping source using high-power pump diode lasers providing the high peak pump power needed for the marker pulse formation and low average pump power between these pulses for loss compensation enables building a pulse-on-demand laser. The losses that have to be compensated in this way are mainly the result of the spontaneous emission, which depletes the inversion population during long pauses between the marker pulses. Because all the control and gain compensation are done by the laser's pump system,

\section{Ider-Marker modulation}
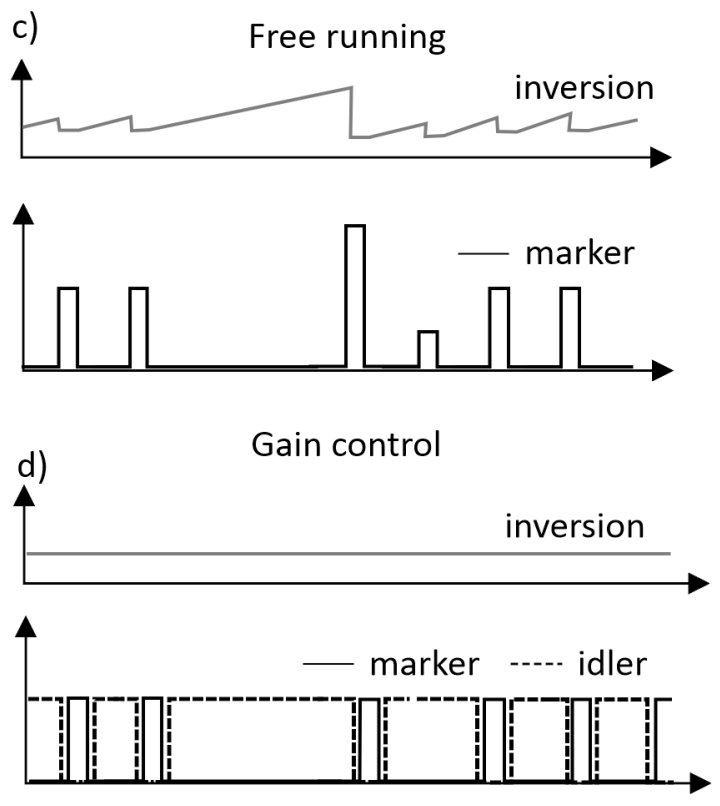

Fig. 1. Comparison of two methods: a) and b) pump-modulation method for high-power oscillator setup, and c) and d) idler-marker seeds for MOPA-based laser systems; in a) and c) the free-running laser output and inversion at arbitrary repetition rate are shown while in b) and d) the corresponding gain control is used 
this enables a compact and cost-effective way of producing pulses on demand when the target marker pulse duration is varied in the nanosecond region.

\subsection{Gain Control via idler and marker Seeds}

For generating picosecond or even femtosecond pulses on demand, gain control using the idler and marker seeds is usually required. Such a laser replaces the pump recovery amplifier with at least one separately pumped amplifying stage. The goal of the idler seed is to prevent the population inversion reaching high values and, as a consequence, gain build-up, which would cause transients when switching on the laser marker signal. The idler is then filtered out after the last amplifying stage.

The combination of two seed-laser diodes with wavelengths separated by $1 \mathrm{~nm}$ was used in a laser setup to generate pulses on demand in the so-called quasi-CW mode of operation. At the output of the laser, a volume Bragg grating was used to filter out the idler signal. The laser is capable of modulating the full $200 \mathrm{~W}$ of output power with a bandwidth of several $10 \mathrm{~s}$ of $\mathrm{MHz}$, producing stable marker pulses with a duration from $50 \mathrm{~ns}$ to $\mathrm{CW}$ [26].

The marker seed-laser diode can be exchanged with a mode-locked laser seeder to expand the marker pulse duration tuning into the ultrashort pulse domain. Here one must take care as the energy of the ultrashort pulses in the last amplifying stage causes unwanted nonlinear effects that can damage the laser system. For this, a cost-effective solution is to upgrade the pulseon-demand laser setup with a solid-state gain crystal that replaces the last power-amplifying stage and shortens the nonlinear interaction length. With this, the threshold for the onset of nonlinear phenomena is greatly increased due to the larger mode field diameter when compared to the fibre power amplifier. This makes possible the production of high-energy picosecond pulses in the pulse-on-demand operation mode.

\section{EXPERIMENTAL}

Three proof-of-concept experimental setups were built to test the operation of the previously mentioned pulseon-demand lasers. A compact gain-switched fibre laser setup covering the $\mathrm{kW}$ peak-power range and nanosecond pulses was built while the advanced setup using a double seeded MOPA fibre laser architecture was used for tests performed over a wider duration tuning range of marker pulses. The latter was also tested on a commercial laser transfer printing system.
Furthermore, the advanced setup was upgraded with a solid-state amplifier and a mode-locked marker seed to generate picosecond pulses on demand.

\subsection{Compact Setup}

A compact pulse-on-demand laser based on a highly adaptable gain-switched fibre laser was realized by using an advanced pump system in a ytterbium-doped fibre oscillator and a pump-recovery amplifier. The oscillator and the pump-recovery amplifier are both pumped by the same pump system and thus retain a low complexity. The advanced pump system consisted of a primary and a secondary pump module. The primary module generated high-power pump pulses with a duration from $500 \mathrm{~ns}$ to $1 \mu \mathrm{s}$ serving as the main energy source for forming the marker pulses in the fibre oscillator. The secondary pump module, operating at a constant pump power of around $1 \mathrm{~W}$, counteracted the losses to the population inversion caused by the spontaneous emission. Switching the pump power from a high to a low value, thus selecting only the first spike of the relaxation oscillation, an arbitrary marker pulse pattern was achieved. The repetition rate could be varied on the fly from a single pulse to above $1 \mathrm{MHz}$. The average power achieved was over $20 \mathrm{~W}$, and the peak power was around $1 \mathrm{~kW}$. The experimental setup shown in Fig. 2 proved the feasibility of a compact pulse-on-demand laser concept and the possibility for further power scaling.

\subsection{Advanced Setup}

The advanced setup used two seed laser diodes (marker and idler), which were wavelength-separated by $1 \mathrm{~nm}$. The marker generated a selected pattern of pulses with a continuously variable repetition rate and pulse duration, while the idler was used to maintain a constant seeding power to the fibre amplifiers at all times, preventing gain build-up between the marker pulses. This made it possible to use a continuous pumping of the amplifiers, preventing the slow response of the pump to affect the overall dynamic performance of the system.

Two fibre amplifiers (Fig. 3) were used to reach $200 \mathrm{~W}$ of output power, and a volume Bragg grating (VBG) was used to filter out the idler signal, which was sent into a beam dump. The laser was capable of modulating the full $200 \mathrm{~W}$ of output power with a bandwidth of several $10 \mathrm{~s}$ of $\mathrm{MHz}$, and both the output power and modulation bandwidth are further scalable; however, in our case, the laser generated stable pulses with $200 \mathrm{~W}$ of power and a duration from a few 10 


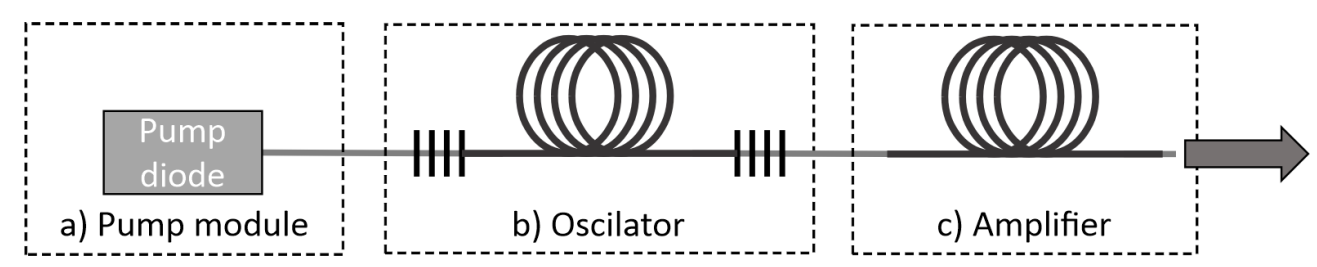

Fig. 2. A compact setup for pulse-on-demand generation based on the gain-switching technique; the pump module a) is used to pump the b) oscillator and c) amplifier; modulation of the pump power in time allows for pulse-on-demand operation

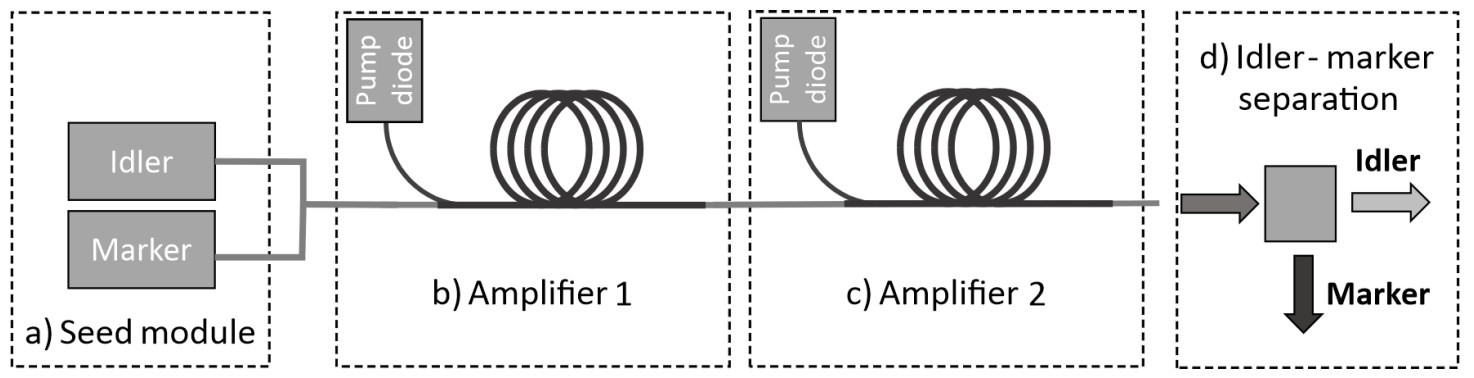

Fig. 3. The advanced system for pulse-on-demand generation; seed module a) consisting of the seed and marker laser diodes keeps the gain balance through the amplifiers b) and c) constant regardless of the marker pulse shape and repetition on the output; the marker and idler signals are separated at the output d) by the volume Bragg grating (VBG)

s of nanoseconds up to continuous $(\mathrm{CW})$ operation at arbitrary repetition rates.

Such laser parameters are useful for many applications, such as thin-film scribing; however, it was designed specifically for LTP in a commercial LTP system, in which the advantage of high modulation bandwidth could be directly evaluated. The printing results are presented in Section 3.1.

\subsection{Advanced Setup - Hybrid Version}

When combining long (nanosecond) idler pulses and short (picosecond or femtosecond) marker pulses, nonlinear filtering can be used to separate idler and marker pulses at the laser output. In our case, second harmonic generation (SHG) was used as a nonlinear filter.

The efficiency of SHG strongly depends on the pulse peak power. Therefore, the efficiency of SHG for long idler pulses with lower peak power is much smaller than the efficiency of SHG for ultrashort marker pulses. In our case, the achieved contrast ratio was 1:3000. The experimental setup for a hybrid pulse-on-demand laser system is shown in Fig. 4.

As already mentioned in the Introduction, nonlinear optical effects can cause problems in ultrashort-fiber laser systems. Due to the small core diameters of the optical fibres, the light intensities in fibre lasers are extremely high. This leads to nonlinear effects such as self-phase modulation
(SPM), which can lead to a shattered pulse temporal profile. To overcome this problem, the chirpedpulse amplification (CPA) technique is used. In CPA systems, the laser pulse is stretched in time prior to amplification. This reduces the pulse peak power in the amplifier and therefore lowers the effect of nonlinear phenomena. The laser pulse is then temporally recompressed outside of the fibre amplifier using a chirped volume Bragg grating (CVBG) or grating compressor.

However, when reaching even higher pulse energies, even CPA systems can no longer achieve pedestal-free pulses at the laser output. To achieve higher energies and a clean temporal profile, an additional solid-state amplifier is added to the fibre amplifying stages. Due to a larger beam diameter in the solid-state amplifier, the nonlinear effects in such an amplifier are almost negligible.

When combining fibre and solid-state amplifiers in pulse-on-demand systems care must be taken to ensure that the idler and marker pulses have the same gain in both fibre and solid-state amplifiers. This is not trivial as the solid-state amplifier has different gain media to the fibre amplifier.

In the fibre amplifier, the gain media has a broad emission spectrum. This means that a broad range of wavelengths around the centre laser wavelength exhibits the same amplification. This is not true in the solid-state gain medium, which has a relatively narrow emission spectrum. Because the spectra of the 


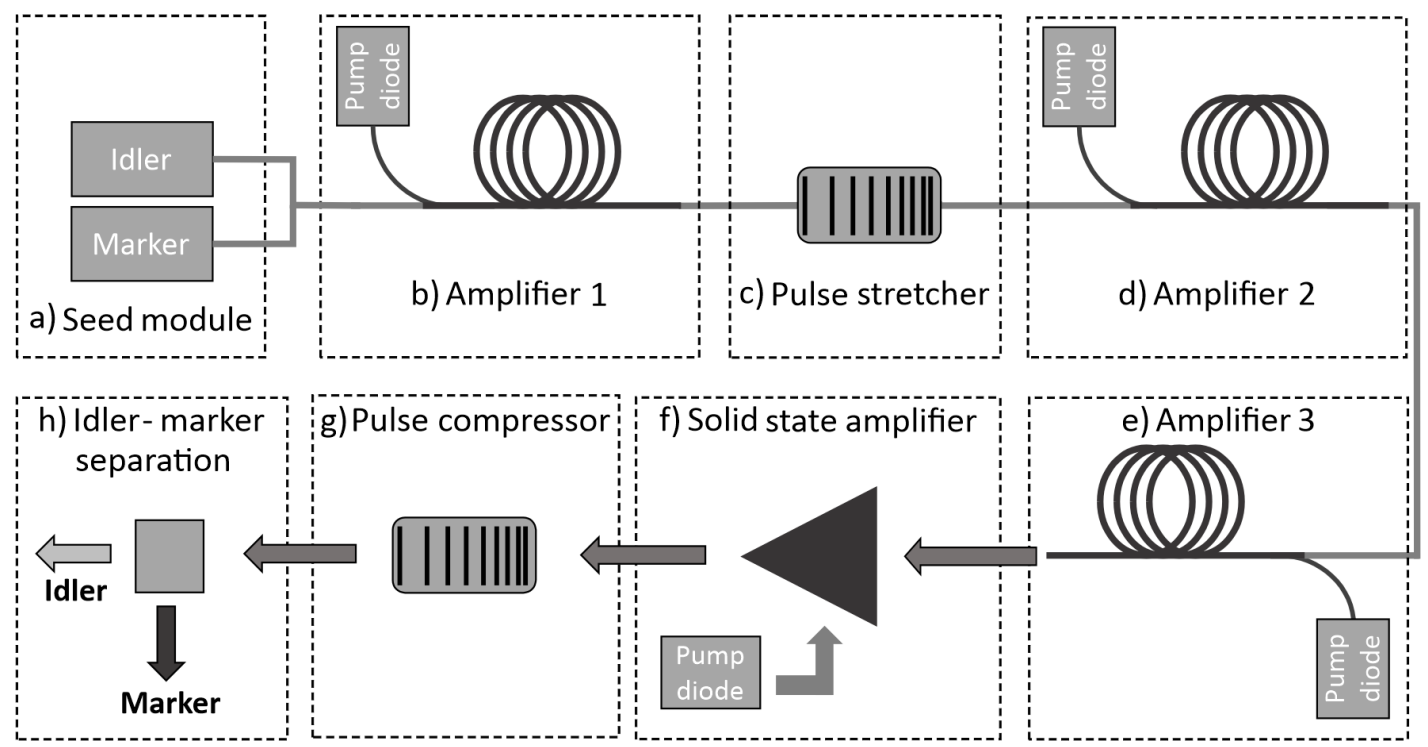

Fig. 4. The advanced hybrid laser system for pulse-on-demand generation; seed module a) where marker seed is exchanged for the mode locked oscillator and b) pre-amplified before it undergoes chirped pulse amplification in the fibre amplifier chain c), d), e); the last amplifying stage $f$ ) is a ytterbium-doped amplifier; the marker - signal separation is made in $h$ ) by the second harmonic generation

idler pulses and marker pulses are not the same, this leads to a different gain for the marker and idler in the solid-state amplifier, whereas the gain is the same for both the idler and marker in the fibre amplifier.

The different gain in the solid-state amplifier leads to transient pulse dynamics when switching from marker to idler pulses and vice versa. To compensate for a different gain in the solid-state amplifier, amplitude modulation of marker and idler pulses is required before amplification. This is similar to the modulation required in burst lasers.

\section{RESULTS}

With the compact gain-switched laser setup a pulse on demand was tested to over $1 \mathrm{MHz}$ repetition rate. In the tested configuration the system could provide pulses tuneable from 40 ns to 100 ns with a peak power of around $1 \mathrm{~kW}$. An arbitrary pulse pattern could be generated. Fig. 5a shows a pulse pattern with the pulse delay changing in a nonlinear way. Such a pattern is required to generate evenly spaced pulses on the workpiece when using a scanner with a nonlinear
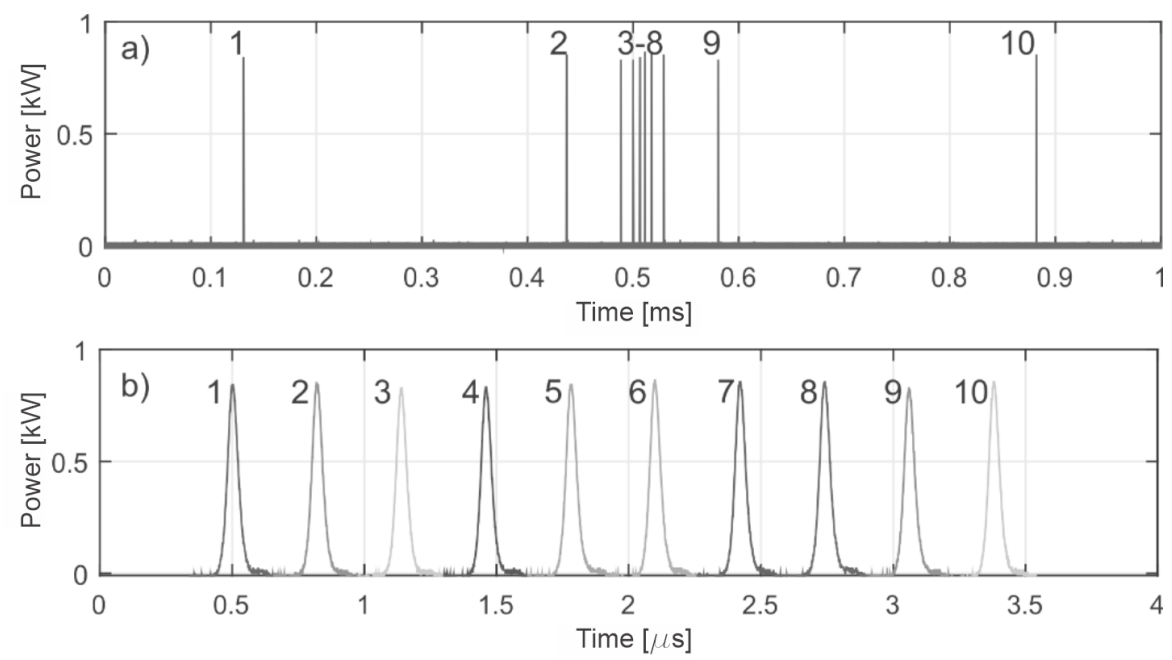

Fig. 5. a) An arbitrary pulse train generated by the compact gain-switched laser setup; b) zoom in on the successive pulses from the trace shown in a); the error in the stability of the pulse width is less than $0.7 \%$ 


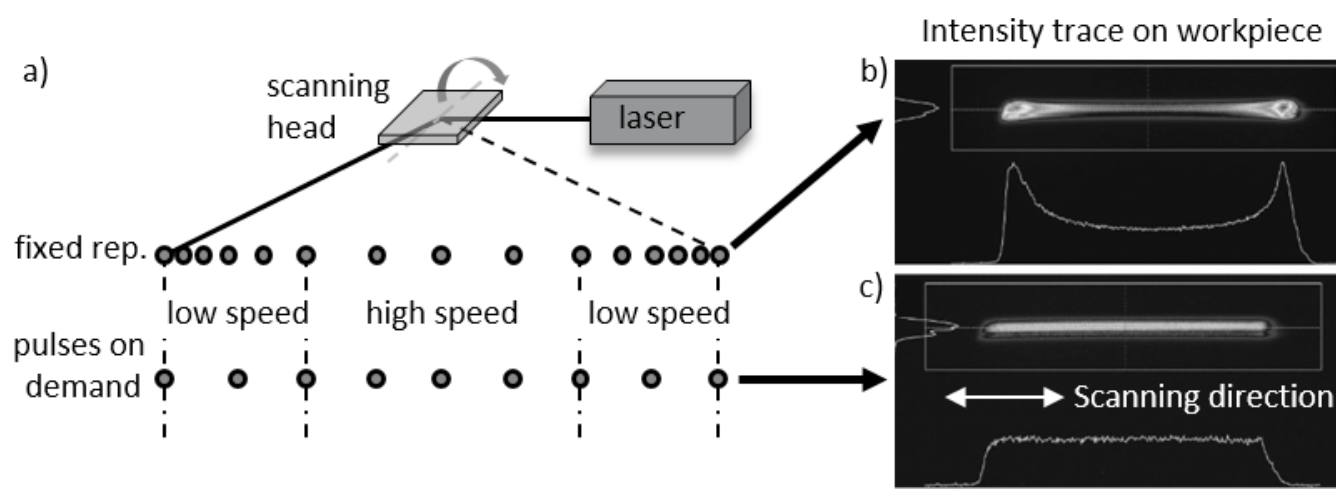

Fig. 6. a) Using a resonant scanner to generate a pattern with laser source operating at a fixed repetition rate captured on the beam profiler camera b); and with the laser source compensating the pulse delay to evenly distribute the laser power over the line trace $c$ )

response function, such as a resonant scanner. A zoom in on the successive pulses in a trace is shown in Fig. $5 \mathrm{~b}$. The delays are omitted on the plot for a better representation of the pulse. The pulse duration was stable with a standard deviation of less than $0.7 \%$, while the peak power fluctuated by less than $1.5 \%$.

The comparison between the laser pulse trace with the nonlinear pulse delay and the pulse trace with the fixed repetition rate made using a resonant scanner is shown in Fig. 6. The intensity distribution for a single trace is shown in Fig. 6b for a fixed repetition rate and Fig. 6c for a pulse-on-demand laser operation synchronized to the resonant scanner. The intensity distribution was captured with the beam-profiler camera. It can be seen that the nonlinear response of the resonant scanner causes more power to be deposited on the trace edges where the scanning speed is low. To make more complex structures, an arbitrary pulse pattern must be used that can compensate for

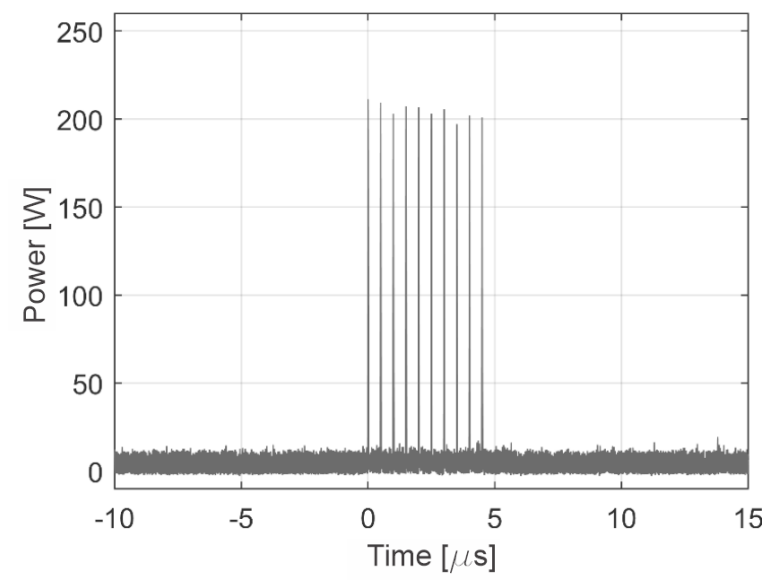

Fig. 7. A typical output (after filtering with a VBG) of the advanced laser; a burst consisting of 10 pulses is shown with a nearly constant power of $200 \mathrm{~W}$ the scanner position in time and still write an arbitrary pulse pattern on the workpiece.

The advanced pulse-on-demand laser system was designed to generate an arbitrary pulse patter with peak power up to $200 \mathrm{~W}$ and modulation of several $10 \mathrm{~s}$ of $\mathrm{MHz}$ to accommodate scanners with higher scanning speeds. A typical laser output (measured with a photodiode) is shown in Fig. 7.

After the marker seed laser diode was exchanged with the mode-locked oscillator and acousto-optics modulator as a low-power pulse picker, the tests were made on the advanced hybrid setup. With appropriate modulation of input idler and marker signal, a stable pulse train with no transient pulse dynamics was achieved when switching between the marker and idler pulses. An oscilloscope trace of a pulse-ondemand sequence after SHG is shown in Fig. 8.

With the presented laser system picosecond pulse-on-demand operation with pulse energies up to $300 \mu \mathrm{J}$ and $450 \mathrm{fs}$ pulse durations were achieved.

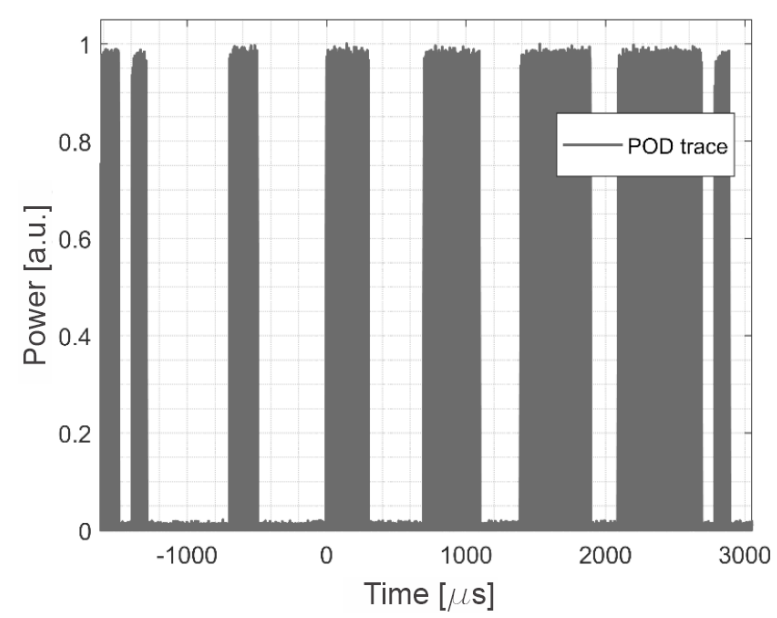

Fig. 8. Pulse-on-demand trace from an advanced hybrid setup (after filtering using the $\mathrm{SHG}$ ) 


\subsection{Laser Transfer-Printing Application}

The advanced laser setup presented in Section 2.2 was tested in a commercial LTP system. In this particular LTP system, the laser beam was focused onto a thin ink film which was spread over a transparent inkcarrier belt, positioned $150 \mu \mathrm{m}$ above the glass acceptor substrate. The energy deposited by the laser heats the ink, which forms a bubble and is finally ejected onto the substrate. The conventional laser used in this LTP system was a $200 \mathrm{~W} \mathrm{CW}$ fibre laser with an external acousto-optical modulator, with a damagethreshold-limited (due to the required tight focusing in the AOM) modulation bandwidth of $5 \mathrm{MHz}$. The beam was scanned using a polygon scanner with a $500 \mathrm{~m} / \mathrm{s}$ scanning speed. The combination of high scanning speed and the limited modulation bandwidth of the conventional laser limits the achievable printing resolution (i.e., the smallest dot that can be transferred onto the substrate).

The advanced pulse-on-demand laser enabled printing with higher resolution or alternatively should, in theory, enable higher printing speeds at the same resolution. The latter would, however, require a faster polygon scanner and was therefore not tested. As for the achieved printing resolution, the laser successfully transferred a single pixel $(1 \mathrm{px})$ sized dot $(1 \mathrm{px}=50$ ns pulse), as seen in Fig. 7, whereas the conventional externally modulated $\mathrm{CW}$ laser only reliably transferred $>4$ px dots (> 200-ns-long pulses).

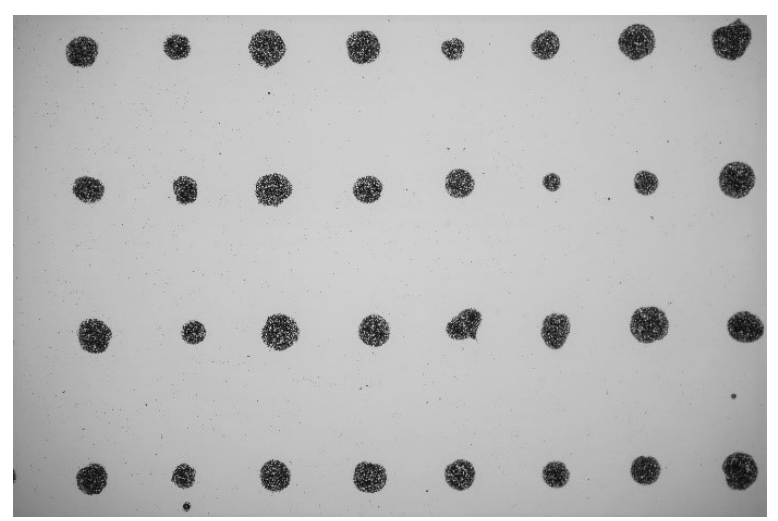

Fig. 9. A series of 1px dots successfully transferred with the advanced laser; the pulse pattern used in this case is shown in Fig. 7; the variations of the printed dot sizes are mostly due to the technical limitations of the LTP process (e.g., ink-thickness variations on the ink-carrier belt)

\section{CONCLUSIONS}

Pulse-on-demand laser operation and its application are presented in this paper. Two methods of efficient gain control that allow for laser pulse parameters to be decoupled from the change in the repetition rate are presented and analysed. The method of direct pump modulation in the high-power laser oscillator was shown to generate pulses on demand from a single shot to repetition rates over $1 \mathrm{MHz}$. The demonstrated result of several tens-of-nanoseconds pulses with a $\mathrm{kW}$ peak power is ideal for applications such as direct colour marking, micromachining and selective photocoagulation.

The advanced method of using a combination of marker and idler seeding in a MOPA (Master Oscillator Power Amplifier) amplifying chain was demonstrated to realize the modulation of a pulse repetition rate of several tens of MHz. The advanced method was tested on an industrial laser transfer-printing system where the synchronization and high modulation bandwidth were evaluated. Furthermore, the advanced method was augmented by a solid-state amplifier providing nonlinear-effect-free operation when the marker seed is producing pulses in the picosecond range.

The benefit of the presented laser systems is that they can be merged into a highly adaptable laser for producing pulses on demand, allowing for precise synchronization with the new generation of highspeed resonant and polygon scanners and thus cover many laser applications.

\section{ACKNOWLEDGEMENTS}

The authors acknowledge the financial support of the Slovenian Research Agency (research core funding No. P2-0270 and projects L2-8183, L2-9240 and L2-9254). The article relates to SPS Operation entitled Building blocks, tools, and systems for future factories-GOSTOP.

\section{REFERENCES}

[1] De Loor, R. (2013). Polygon scanner system for ultra short pulsed laser micro-machining applications. Physics Procedia, vol. 41, p. 544-551, D0I:10.1016/J.phpro.2013.03.114.

[2] Harth, F., Piontek, M.C., Herrmann, T., L'huillier, J.A. (2016). Ultra high-speed micromachining of transparent materials using high PRF ultrafast lasers and new resonant scanning systems. Proceedings SPIE (vol. 9736) Laser-Based Microand Nanoprocessing X, 97360N, D0I:10.1117/12.2212816.

[3] Mur, J., Kavčič, B., Poberaj, I. (2013). Fast and precise Laguerre-Gaussian beam steering with acousto-optic deflectors. Applied Optics, vol. 52, no. 26, p. 6506-6511, DOI:10.1364/A0.52.006506.

[4] Mur, J., Podobnik, B., Poberaj, I. (2017). Laser beam steering approaches for microstructuring of copper layers. Optics 
\& Laser Technology, vol. 88, p. 140-146, D0I:10.1016/j. optlastec.2016.08.009.

[5] Šajn, M., Petelin, J., Agrež, V., Vidmar, M., Petkovšek, R. (2017). DFB diode seeded low repetition rate fiber laser system operating in burst mode. Optics \& Laser Technology, vol. 88, p. 99-103, D0I:10.1016/j.optlastec.2016.09.006.

[6] Bollig, C., Hofmeister, P.-G., Kunze, M., Schmidt, J., Fayed, S., Reuter, R. (2013). Efficient single-frequency pulsed all-fibre amplifier for coherent lidar. Conference on Lasers and ElectroOptics - International Quantum Electronics Conference, DOI:10.1109/CLEOE-IQEC.2013.6801383.

[7] Klehr, A., Liero, A., Wenzel, H., Bugge, F., Brox, O., Fricke, J., Ressel, P., Knigge, A., Heinrich, W., Tränkle, G. (2017). 1030$\mathrm{nm}$ diode-laser-based light source delivering pulses with nanojoule energies and picosecond duration adjustable by mode locking or pulse gating operation. Novel InPlane Semiconductor Lasers XVI (vol. 10123), 101230D, DOl:10.1117/12.2250093.

[8] Petkovšek, R., Agrež, V., Bammer, F., Jakopič, P., Lenardič, B. (2013). Experimental and theoretical study of gain switched Yb-doped fiber laser. Proceedings Fiber Lasers X: Technology, Systems, and Applications (vol. 8601), 860128, p. 860128860128, DOl:10.1117/12.2002324.

[9] Agrež, V., Petkovšek, R. (2013). Gain-switched Yb-doped fiber laser for microprocessing. Applied Optics, vol. 52, no. 13, p. 3066-3072, DOI:10.1364/A0.52.003066.

[10] Yang, J., Tang, Y., Xu, J. (2013). Development and applications of gain-switched fiber lasers [Invited]. Photonics Research, vol. 1, no. 1, p. 52-57, D0I:10.1364/PRJ.1.000052.

[11] Agrež, V., Petkovšek, R. (2014). Gain switch laser based on micro-structured Yb-doped active fiber. Optics Express, vol. 22, no. 5, p. 5558-5563, D0I:10.1364/0E.22.005558.

[12] Petkovšek, R., Agrež, V. (2014). Single stage Yb-doped fiber laser based on gain switching with short pulse duration. Optics Express, vol. 22, no. 2, p. 1366-1371, Dol:10.1364/ 0E.22.001366.

[13] Petkovšek, R., Agrež, V., Sangla, D., Saby, J., Picard, R.B., Salin, F. (2014). Gain-switched ytterbium-doped rod-type fiber laser. Laser Physics Letters, vol. 11, no. 10, p. 105808, DOI:10.1088/1612-2011/11/10/105808.

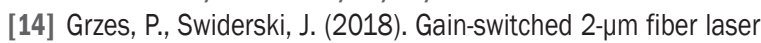
system providing kilowatt peak-power mode-locked resembling pulses and its application to supercontinuum generation in fluoride fibers. IEEE Photonics Journal, vol. 10, no. 1, p. 1-8, DOI:10.1109/JPHOT.2018.2791634.

[15] Moulton, P.F., Rines, G.A., Slobodtchikov, E.V., Wall, K.F., Frith, G., Samson, B., Carter, A.L.G. (2009). Tm-doped fiber lasers: fundamentals and power scaling. IEEE Journal of Selected Topics in Quantum Electronics, vol. 15, no. 1, p. 85-92, DOI:10.1109/jstqe.2008.2010719.

[16] Šušnjar, P., Agrež, V., Petkovšek, R. (2018). Photodarkening as a heat source in ytterbium doped fiber amplifiers. Optics Express, vol. 26, no. 5, p. 6420-6426, Dol:10.1364/ 0E.26.006420.

[17] Luo, H., Liu, F., Li, J., Liu, Y. (2018). High repetition rate gain-switched Ho-doped fiber laser at $2.103 \mu \mathrm{m}$ pumped by h-shaped mode-locked Tm-doped fiber laser at 1.985 «m. Optics Express, vol. 26, no. 20, p. 26485-26494, DOI:10.1364/0E.26.026485.

[18] Paradis, P., Fortin, V., Aydin, Y.O., Vallée, R., Bernier, M. (2018). $10 \mathrm{~W}$-level gain-switched all-fiber laser at $2.8 \mu \mathrm{m}$. Optics Letters, vol. 43, no. 13, p. 3196-3199, Dol:10.1364/ 0L.43.003196.

[19] Mur, J., Petelin, J., Osterman, N., Petkovšek, R. (2017). High precision laser direct microstructuring system based on bursts of picosecond pulses. Journal of Physics D: Applied Physics, vol. 50, no. 32, ID 325104, D0l:10.1088/1361-6463/aa7b5a.

[20] Mur, J., Pirker, L., Osterman, N., Petkovšek, R. (2017). Silicon crystallinity control during laser direct microstructuring with bursts of picosecond pulses. Optics Express, vol. 25, no. 21, p. 26356-26364, D0I:10.1364/0E.25.026356.

[21] Mur, J., Petkovšek, R. (2019). Near-THz bursts of pulses Governing surface ablation mechanisms for laser material processing. Applied Surface Science, vol. 478, p. 355-360, DOI:10.1016/j.apsusc.2019.01.182.

[22] Černe, L., Novak, J., Agrež, V., Petkovšek, R. (2019). Optimization of a supercontinuum source based on tapered ordinary fibers. Laser Physics, vol. 29, no. 2, ID 025103. DOI:10.1088/1555-6611/aaf640.

[23] Motoshima, K., Leba, L. M., Chen, D.N., Downs, M.M., Li, T., Desurvire, E. (1993). Dynamic compensation of transient gain saturation in erbium-doped fiber amplifiers by pump feedback control. IEEE Photonics Technology Letters, vol. 5, no. 12, p. 1423-1426, D0I:10.1109/68.262563.

[24] Novak, V., Podobnik, B., Možina, J., Petkovšek, R. (2013). Analysis of the thermal management system for a pump laser. Applied Thermal Engineering, vol. 57, no. 1-2, p. 99-106, DOI:10.1016/j.applthermaleng.2013.03.060.

[25] Harth, F., Herrmann, T., L'huillier, J.A. (2019). High power ultrafast laser with highly dynamic repetition rate and constant pulse energy from single pulse to $10 \mathrm{MHz}$. Solid State Lasers XXVIII: Technology and Devices (vol. 10896), 108960H, DOl:10.1117/12.2507821.

[26] Petkovšek, R., Novak, V., Agrež, V. (2015). High power fiber MOPA based QCW laser delivering pulses with arbitrary duration on demand at high modulation bandwidth. Optics Express, vol. 23, no. 26, p. 33150-33156, D0l:10.1364/ OE.23.033150.

[27] Petkovšek, R., Novak, V., Agrež, V. (2017). Fiber laser for high speed laser transfer printing. Proceedings of XXI International Symposium on High Power Laser Systems and Applications, vol. 10254, ID 1025403, DOI:10.1117/12.2257862.

[28] Tuennermann, A., Zellmer, H., Ruske, J.P. (2001). Directly Modulatable Laser. US 20010014107, US Patent and Trademark Office, Washington.

[29] Quentin, U., Kanal, F., Budnicki, A., Tan, C., Diekamp, H., Jansen, F., Scelle, R., Sutter, D. (2019). TruMicro 2000: Nextgeneration flexible ultrashort pulse fiber lasers for scientific and industrial applications (Conference Presentation). Proceedings of Fiber Lasers XVI: Technology and Systems (vol. 10897), 108971M, D0I:10.1117/12.2511120.

[30] Munroe, M.J. (2011). Method and Apparatus for Producing Arbitrary Pulsetrains from a Harmonic Fiber Laser, US 7885298, US Patent and Trademark Office, Washington. 
[31] Elahi, P., Yılmaz, S., Eldeniz, Y.B., Ilday, F.Ö. (2014). Generation of picosecond pulses directly from a $100 \mathrm{~W}$, burst-mode, doping-managed Yb-doped fiber amplifier. Optics Letters, vol. 39, no. 2, p. 236-239, DOl:10.1364/0L.39.000236.

[32] Petelin, J., Podobnik, B., Petkovšek, R. (2015). Burst shaping in a fiber-amplifier chain seeded by a gain-switched laser diode. Applied Optics, vol. 54, no. 15, p. 4629-4634, D0l:10.1364/ A0.54.004629.

[33] Agrež, V., Petkovšek, R., Sangla, D., Saby, J., Picard, R.B.; Salin, F. (2014). Effect of repetition rate on gain-switched fiber laser output pulses. Laser Physics, vol. 24, no. 10, ID 105108 , DOI:10.1088/1054-660x/24/10/105108.

[34] Petelin, J., Agrež, V., Podobnik, B., Petkovšek, R. (2014). Short pulsed gain-switched fiber laser with improved efficiency utilizing unabsorbed pump recovery. Optics Express, vol. 22, no. 17, p. 20588-20594, DOl:10.1364/0E.22.020588.

[35] Agrež, V., Petkovšek, R. (2019). Highly adaptable gainswitched fiber laser with improved efficiency. Optics Express, vol. 27, no. 9, p. 12100-12109, D0I:10.1364/0E.27.012100. 